PROCEEDINGS OF THE

AMERICAN MATHEMATICAL SOCIETY

Volume 126, Number 2, February 1998, Pages 481-489

S 0002-9939(98)04079-9

\title{
STABILITY OF WEAKLY ALMOST CONFORMAL MAPPINGS
}

\author{
BAISHENG YAN AND ZHENGFANG ZHOU
}

(Communicated by Albert Baernstein II)

\begin{abstract}
We prove a stability of weakly almost conformal mappings in $W^{1, p}\left(\Omega ; \mathbf{R}^{n}\right)$ for $p$ not too far below the dimension $n$ by studying the $W^{1, p_{-}}$ quasiconvex hull of the set $\mathcal{C}_{n}$ of conformal matrices. The study is based on coercivity estimates from the nonlinear Hodge decompositions and reverse Hölder inequalities from the Ekeland variational principle.
\end{abstract}

\section{INTRODUCTION AND MAIN RESULTS}

Let $\mathcal{M}^{n \times n}$ be the set of all real $n \times n$ matrices. Let $X: Y=\operatorname{tr}\left(X^{T} Y\right)$ and $|X|^{2}=X: X$ for $X, Y \in \mathcal{M}^{n \times n}$, where $A^{T}$ and $\operatorname{tr} A$ denote the transpose and the trace of $A$, respectively. Note that Hadamard's inequality implies $|A|^{n} \geq n^{n / 2} \operatorname{det} A$ for all matrices $A$. For $l \geq 1$, we define a set $K_{l}$ by

$$
K_{l}=\left\{\left.A \in \mathcal{M}^{n \times n}|| A\right|^{n} \leq l n^{n / 2} \operatorname{det} A\right\} .
$$

If $l=1$ then $K_{1}$ coincides with the conformal set $\mathcal{C}_{n}=\{\lambda Q \mid \lambda \geq 0, Q \in S O(n)\}$, where $S O(n)$ is the set of all orthogonal matrices $X$ with $\operatorname{det} X=1$.

The set $K_{l}$ is related to the theory of $l$-quasiregular mappings (see Iwaniec [11] and Reshetnyak [16]). Let $\Omega \subset \mathbf{R}^{n}$ be a domain and $u \in W_{l o c}^{1, p}\left(\Omega ; \mathbf{R}^{n}\right)$. Denote by $\nabla u(x)$ the gradient matrix

$$
(\nabla u(x))_{i j}=\partial u^{i}(x) / \partial x_{j}, \quad 1 \leq i, j \leq n .
$$

A map $u \in W_{l o c}^{1, p}\left(\Omega ; \mathbf{R}^{n}\right)$ is called (weakly if $p<n$ ) $l$-quasiregular if $\nabla u(x) \in K_{l}$ for a.e. $x \in \Omega$. A (weakly) 1-quasiregular map is also called a (weakly) conformal map.

A significant result of Iwaniec [11] (see also [13]) shows that for each $n \geq 3$ and $l \geq 1$ there exists a number $p_{*}<n$ such that every weakly $l$-quasiregular map in $W_{l o c}^{1, p_{*}}\left(\Omega ; \mathbf{R}^{n}\right)$ belongs actually to $W_{l o c}^{1, n}\left(\Omega ; \mathbf{R}^{n}\right)$. Given $n \geq 3$, for each $l \geq 1$, let $p_{*}(l)$ be the infimum of all such numbers $p_{*}<n$ as described above. If the dimension $n$ is even, Iwaniec and Martin [12] has showed that $p_{*}(1)=n / 2$. A general conjecture posed in Iwaniec [11] is that $p_{*}(l)=\frac{n l}{l+1}$ (see also [12]).

To discuss our main results, we first review some important notation and preliminaries in the calculus of variations that will be needed in this paper.

Let $f: \mathcal{M}^{n \times n} \rightarrow \mathbf{R}$ be a function. Denote by $\mathcal{Z}(f)$ the zero set of $f$. Define the quasiconvexification $f^{q c}$ of $f$ by

$$
f^{q c}(A)=\inf _{\phi \in C_{0}^{\infty}\left(\Omega ; \mathbf{R}^{n}\right)} f_{\Omega} f(A+\nabla \phi(x)) d x, \quad A \in \mathcal{M}^{n \times n},
$$

Received by the editors February 26, 1996 and, in revised form, August 12, 1996. 1991 Mathematics Subject Classification. Primary 49J10, 35J50, 30C62.

(C) 1998 American Mathematical Society 
where $f_{D} g$ denotes the integral average of $g$ over the domain $D$, i.e.,

$$
f_{D} g(x) d x=\frac{1}{|D|} \int_{D} g(x) d x
$$

A simple argument using Vitali's covering shows that $f^{q c}(A)$ is independent of domain $\Omega$. We say that $f$ is quasiconvex on $\mathcal{M}^{n \times n}$ provided that $f^{q c} \equiv f$. If $f$ is continuous and satisfies the growth condition (1.3) below then $f^{q c}$ is continuous and quasiconvex, and in this case for each measurable set $S \subseteq \Omega$ the functional $\Phi_{S}(u)=\int_{S} f^{q c}(\nabla u)$ is sequentially weakly lower semicontinuous on $W^{1, p}\left(\Omega ; \mathbf{R}^{n}\right)$. We refer to Acerbi-Fusco [1], Ball [2], Ball-Murat [3], Dacorogna [4] and Morrey [14] for proofs and further information.

Let $\mathcal{K} \subseteq \mathcal{M}^{n \times n}$ and $1 \leq p \leq \infty$ be given. Let $\mathcal{Q}_{p}^{+}(\mathcal{K})$ be the set of all continuous quasiconvex functions $f$ on $\mathcal{M}^{n \times n}$ satisfying that $\mathcal{K} \subseteq \mathcal{Z}(f)$ and

$$
0 \leq f(X)<C\left(|X|^{p}+1\right) \quad \forall X \in \mathcal{M}^{n \times n} .
$$

Then the $W^{1, p}$-quasiconvex hull $\mathbf{Q}_{p}(\mathcal{K})$ of $\mathcal{K}$ is defined by

$$
\mathbf{Q}_{p}(\mathcal{K})=\bigcap\left\{\mathcal{Z}(f) \mid f \in \mathcal{Q}_{p}^{+}(\mathcal{K})\right\} .
$$

We refer to Yan [20] for general theory and applications of quasiconvex hulls $\mathbf{Q}_{p}(\mathcal{K})$.

It has been proved $([17],[20],[21])$ that $\mathbf{Q}_{\infty}(\mathcal{K})=\mathbf{Q}_{1}(\mathcal{K})$ for all bounded sets $\mathcal{K}$, thus the $W^{1, p}$-quasiconvex hulls of a bounded set are the same for all $1 \leq p \leq \infty$. For unbounded sets, however, $W^{1, p}$-quasiconvex hulls may depend remarkably on values of $p \geq 1$. For instance, the following has been proved in Müller-Šverák-Yan [15] and Yan [19].

Theorem A. Let $n \geq 3$. Then $\mathbf{Q}_{p}\left(\mathcal{C}_{n}\right)=\mathcal{C}_{n}$ for $p \geq n$, while $\mathbf{Q}_{p}\left(\mathcal{C}_{n}\right)=\mathcal{M}^{n \times n}$ for $1 \leq p<n / 2$. Moreover, if $n$ is even then $\mathbf{Q}_{p}\left(\mathcal{C}_{n}\right)=\mathcal{C}_{n}$ for $p \geq n / 2$.

In this paper, we shall study the $W^{1, p}$-quasiconvex hull $\mathbf{Q}_{p}\left(\mathcal{C}_{n}\right)$ for $n / 2 \leq p<n$ when $n \geq 3$ is odd. We shall prove the following main theorem.

Theorem B. For $n \geq 3$ there exists a $p<n$ such that $\mathbf{Q}_{p}\left(\mathcal{C}_{n}\right) \equiv \mathcal{C}_{n}$. Moreover, if $p_{0}(n)$ denotes the infimum of all such $p$, then $p_{0}(n) \geq n / 2$.

Remark. First, the second part of the theorem follows directly from Theorem A. Secondly, if $n$ is even, $p_{0}(n)=n / 2$ and is also the minimum; however, we do not know whether $p_{0}(n)=n / 2$ if $n$ is odd. Finally, the following stability result on weakly almost conformal mappings can be derived from Theorem B (see [20, Theorem 3.1]).

Corollary C. Let $n \geq 3$, and let $\left\{u_{j}\right\}$ in $W^{1, p}\left(\Omega ; \mathbf{R}^{n}\right)$ be a sequence of (weakly if $p<n)$ almost conformal mappings in the sense that $\int_{\Omega} d_{\mathcal{C}_{n}}^{p}\left(\nabla u_{j}\right) \rightarrow 0$ as $j \rightarrow$ $\infty$, where $d_{\mathcal{C}_{n}}(X)$ is the distance from $X$ to $\mathcal{C}_{n}$. If $u_{j} \rightarrow u_{0}$ in $W^{1, p}\left(\Omega ; \mathbf{R}^{n}\right)$ and $p_{0}(n)<p \leq n$, then the weak limit $u_{0}$ is weakly conformal.

\section{Nonlinear Hodge Decompositions}

One of the key tools for proving the main theorem is the following stability result on nonlinear Hodge decompositions due to Greco-Iwaniec [10] (see also [11], [13]). 
Lemma 2.1. Let $n \geq 3$ and $n-\frac{1}{2} \leq p \leq n$, and let $\mathbf{B}=B(a, R)$ be a ball in $\mathbf{R}^{n}$ with center $a$ and radius $R$. Then for each $u \in W^{1, p}\left(\mathbf{B} ; \mathbf{R}^{n}\right)$ the matrix field $|\nabla u|^{p-n} \nabla u \in L^{\frac{p}{1+p-n}}\left(\mathbf{B} ; \mathcal{M}^{n \times n}\right)$ can be decomposed as

$$
|\nabla u(x)|^{p-n} \nabla u(x)=\nabla \psi(x)+h(x) \quad \text { a.e. } x \in \mathbf{B},
$$

where $\psi \in W^{1, \frac{p}{1+p-n}}\left(\mathbf{R}^{n} ; \mathbf{R}^{n}\right)$ and $h \in L^{\frac{p}{1+p-n}}\left(\mathbf{R}^{n} ; \mathcal{M}^{n \times n}\right)$ is a divergence free matrix field such that

$$
\|h\|_{L^{\frac{p}{1+p-n}}\left(\mathbf{R}^{n}\right)} \leq(n-p) \beta_{n}\|\nabla u\|_{L^{p}(\mathbf{B})}^{1+p-n} .
$$

Proof. Decomposition (2.1) follows from Greco-Iwaniec [10, Lemma 2.1] with $\epsilon=$ $n-p \in\left[0, \frac{1}{2}\right]$. In this case, we have

$$
\left(\int_{\mathbf{R}^{n}}|h(x)|^{\frac{n-\epsilon}{1-\epsilon}} d x\right)^{\frac{1-\epsilon}{n-\epsilon}} \leq A(n, n-\epsilon) \epsilon\left(\int_{\mathbf{B}}|\nabla u(x)|^{n-\epsilon} d x\right)^{\frac{1-\epsilon}{n-\epsilon}} .
$$

As seen in the arguments of Greco-Iwaniec [10, Lemma 2.1] and Iwaniec-Sbordone $[13,(1.13)$ and $(2.10)]$, the constant $A(n, n-\epsilon)$ in (2.3) can be chosen independent of $\epsilon$ if $0 \leq \epsilon \leq 1 / 2$. The proof is completed.

We need a similar decomposition for mappings with affine boundary condition.

Proposition 2.2. Let $n-\frac{1}{2} \leq p \leq n$ and $u \in W^{1, p}\left(\mathbf{B} ; \mathbf{R}^{n}\right)$ satisfy $\left.u\right|_{\partial \mathbf{B}}=A x$. Then we have decompositions

$$
|\nabla u(x)|^{p-n} \nabla u(x)=\nabla \Psi(x)+H(x) \quad \text { a.e. } x \in \mathbf{B}
$$

which satisfy

$$
f_{\mathbf{B}} \nabla \Psi=|A|^{p-n} A, \quad\|H\|_{L^{\frac{p}{1+p-n}}(\mathbf{B})} \leq(n-p) \alpha_{n}\|\nabla u\|_{L^{p}(\mathbf{B})}^{1+p-n} .
$$

Proof. For simplicity, let $q=\frac{p}{1+p-n}$ and $q^{*}$ be the Sobolev index of $q$, i.e., $\left(q^{*}\right)^{-1}=$ $q^{-1}-n^{-1}$. Let $\mathbf{B}=\mathbf{B}_{r}$ with radius $r>0$. Extend $u(x)$ by $A x$ outside $\mathbf{B}_{r}$ and apply Lemma 2.1 to $u \in W^{1, p}\left(\mathbf{B}_{3 r} ; \mathbf{R}^{n}\right)$; we have

$$
|\nabla u(x)|^{p-n} \nabla u(x)=\nabla \psi(x)+h(x) \quad \text { a.e. } x \in \mathbf{B}_{3 r} .
$$

Since $\nabla u \equiv A$ outside $\mathbf{B}_{r}$, we easily have $\|\nabla u\|_{L^{p}\left(\mathbf{B}_{3 r}\right)} \leq c_{n}\|\nabla u\|_{L^{p}\left(\mathbf{B}_{r}\right)}$. Thus using $(2.2)$ on $\mathbf{B}_{3 r}$ we have

$$
\|h\|_{L^{q}\left(\mathbf{R}^{n}\right)} \leq(n-p) \beta_{n}^{\prime}\|\nabla u\|_{L^{p}\left(\mathbf{B}_{r}\right)}^{1+p-n} .
$$

Define $\Psi(x)=\psi(x)+A_{0} x$ and $H(x)=h(x)-A_{0}$, where $A_{0}=|A|^{p-n} A-f_{\mathbf{B}_{r}} \nabla \psi$ so that $f_{\mathbf{B}_{r}} \nabla \Psi=|A|^{p-n} A$. Then (2.4) follows easily. We need to estimate $\|H\|_{L^{q}\left(\mathbf{B}_{r}\right)}$. To this end, let $f(x)=|A|^{p-n} A x-\psi(x)+\gamma$ and $\gamma$ be the constant making $\int_{D} f=0$, where $D=\mathbf{B}_{3 r} \backslash \mathbf{B}_{r}$. It is easy to see that $f$ is both harmonic and satisfies $\nabla f=h$ in $D$. Hence, for any $z \in \partial \mathbf{B}_{2 r}$, using the mean value theorem of harmonic functions, we deduce by the Hölder and Sobolev-Poincaré inequalities

$$
\begin{aligned}
|f(z)| & =\left|f_{\mathbf{B}(z, r)} f\right| \leq c_{n} r^{-\frac{n}{q^{*}}}\|f\|_{L^{q^{*}}(D)} \\
& \leq c_{n} r^{-\frac{n}{q^{*}}}\|\nabla f\|_{L^{q}(D)} \leq c_{n} r^{1-\frac{n}{q}}\|h\|_{L^{q}\left(\mathbf{R}^{n}\right)} .
\end{aligned}
$$


Note that $\left|\mathbf{B}_{r}\right| A_{0}=\int_{\mathbf{B}_{r}} \nabla f=\int_{\mathbf{B}_{2 r}} \nabla f-\int_{\mathbf{B}_{2 r} \backslash \mathbf{B}_{r}} h$. Thus by Hölder's inequality, second term on the right-hand side can be estimated as

$$
\left|\int_{\mathbf{B}_{2 r} \backslash \mathbf{B}_{r}} h\right| \leq c_{n} r^{n\left(1-\frac{1}{q}\right)}\|h\|_{L^{q}\left(\mathbf{R}^{n}\right)} ;
$$

while the first term by using the divergence theorem and (2.8) can be estimated as

$$
\left|\int_{\mathbf{B}_{2 r}} \nabla f\right|=\left|\int_{\partial \mathbf{B}_{2 r}} f \otimes \vec{n}\right| \leq c_{n} r^{n\left(1-\frac{1}{q}\right)}\|h\|_{L^{q}\left(\mathbf{R}^{n}\right)},
$$

where $\vec{n}$ is the unit normal vector on the boundary. Therefore, we obtain

$$
\left|A_{0}\right| \leq c_{n} r^{-\frac{n}{q}}\|h\|_{L^{q}\left(\mathbf{R}^{n}\right)}, \quad\left\|A_{0}\right\|_{L^{q}\left(\mathbf{B}_{r}\right)} \leq c_{n}\|h\|_{L^{q}\left(\mathbf{R}^{n}\right)} .
$$

Since $H=h-A_{0}$, we have

$$
\|H\|_{L^{q}\left(\mathbf{B}_{r}\right)} \leq\|h\|_{L^{q}\left(\mathbf{B}_{r}\right)}+\left\|A_{0}\right\|_{L^{q}\left(\mathbf{B}_{r}\right)} \leq c_{n}\|h\|_{L^{q}\left(\mathbf{R}^{n}\right)},
$$

which combined with (2.7) yields the second estimate on $H$ in (2.5). The proof is thus completed.

Let

$$
p_{1}(n)=\max \left\{n-\frac{1}{2}, n-\frac{1}{2 \alpha_{n}}\right\}, \quad l(p)=\frac{1}{1-(n-p) \alpha_{n}} .
$$

We shall also need a regularity result on quasiregular mappings based on the work of Iwaniec [11].

Lemma 2.3. There exists a $p_{2}(n) \in\left[p_{1}(n), n\right)$ such that if $u \in W_{l o c}^{1, p}\left(\Omega ; \mathbf{R}^{n}\right)$ and $\nabla u(x) \in K_{l(p)}$ for some $p \geq p_{2}(n)$ then $u \in W_{l o c}^{1, n}\left(\Omega ; \mathbf{R}^{n}\right)$.

Proof. Let $p_{*}(l)$ be as defined in the introduction. Then it is easily seen that $p_{*}(l)$ is nondecreasing, thus the function $\mu(p)=p-p_{*}(l(p))$ is increasing on $\left[p_{1}(n), n\right)$. Since $\mu(p) \rightarrow n-p_{*}\left(1^{+}\right)>0$ as $p \rightarrow n^{-}$, hence there exists $p_{2}(n) \in\left[p_{1}(n), n\right)$ such that $\mu(p)>0$, i.e., $p>p_{*}(l(p))$, for all $p_{2}(n) \leq p \leq n$. Therefore, by definition of $p_{*}(l)$, we deduce the conclusion of the lemma.

\section{Coercivity estimates Below the Dimension}

In this section, we prove some coercivity estimates for conformal energy functionals (see Yan [19]) in $W^{1, p}\left(\Omega ; \mathbf{R}^{n}\right)$ for $p<n$.

Theorem 3.1. Let $f$ be a nonnegative continuous function which is homogeneous of degree 1 and satisfies $\mathcal{Z}(f)=\mathcal{C}_{n}$. Then for any ball $\mathbf{B}$ and $p \in\left[n-\frac{1}{2}, n\right]$ and $\delta>0$ there exists a constant $C_{f}(n, \delta)$ such that

$$
\begin{aligned}
& {\left[1-(n-p) \alpha_{n}-\delta\right] f_{\mathbf{B}}|A+\nabla \phi(x)|^{p} d x-n^{\frac{n}{2}}|A|^{p-n} \operatorname{det} A} \\
& \leq C_{f}(n, \delta) f_{\mathbf{B}} f^{p}(A+\nabla \phi(x)) d x, \quad A \in \mathcal{M}^{n \times n}, \phi \in C_{0}^{\infty}\left(\mathbf{B} ; \mathbf{R}^{n}\right) .
\end{aligned}
$$

Proof. We prove (3.1) first for a special function $f=h$, then the general case will follow from homogeneity (see [15]). To this end, consider $F: \mathcal{M}^{n \times n} \rightarrow \mathcal{M}^{n \times n}$ defined by

$$
F(X)=|X|^{n-2} X-n^{\frac{n-2}{2}}(\operatorname{adj} X)^{T}
$$


where $\operatorname{adj} X$ is the adjugate matrix of $X$, i.e., $X(\operatorname{adj} X)=(\operatorname{det} X) I$ with $I$ being the identity matrix. Let $h(X)=|F(X)|^{\frac{1}{n-1}}$. Then $h$ is continuous and homogeneous of degree 1 and satisfies $\mathcal{Z}(h)=\mathcal{C}_{n}$. We now prove the theorem for $f=h$.

For $A \in \mathcal{M}^{n \times n}, \phi \in C_{0}^{\infty}\left(\mathbf{B} ; \mathbf{R}^{n}\right)$, let $u(x)=A x+\phi(x)$ and let $\Psi, H$ be as given in (2.4). Note that

$$
F(\nabla u): \nabla \Psi=|\nabla u|^{p}-|\nabla u|^{n-2} \nabla u: H-n^{\frac{n-2}{2}}(\operatorname{adj} \nabla u)^{T}: \nabla \Psi,
$$

thus using $\operatorname{div}\left[(\operatorname{adj} \nabla u)^{T}\right]=0$ and the divergence theorem, we have by $(2.5)$

$$
\int_{\mathbf{B}} n^{\frac{n-2}{2}}(\operatorname{adj} \nabla u)^{T}: \nabla \Psi=n^{\frac{n-2}{2}}(\operatorname{adj} A)^{T}: \int_{\mathbf{B}} \nabla \Psi=n^{\frac{n}{2}}|A|^{p-n} \operatorname{det} A|\mathbf{B}| .
$$

Integrating (3.2) over B and using estimates in (2.5) and Young's inequality, we obtain

$$
\begin{gathered}
{\left[1-(n-p) \alpha_{n}\right] f_{\mathbf{B}}|\nabla u|^{p}-n^{\frac{n}{2}}|A|^{p-n} \operatorname{det} A \leq f_{\mathbf{B}}|\nabla \Psi||F(\nabla u)|} \\
\leq \delta f_{\mathbf{B}}|\nabla u|^{p}+C(n, \delta) f_{\mathbf{B}} h^{p}(\nabla u),
\end{gathered}
$$

which proves (3.1). The proof is completed.

Let $l(p)$ and $p_{2}(n)$ be defined by $(2.10)$. Then $1 \leq l(p) \leq 2$ for $p \in\left[p_{2}(n), n\right]$. In (3.1), note that $f_{\mathbf{B}}|A+\nabla \phi|^{p} \geq|A|^{p}$, and thus by (1.2) we have

$$
\begin{aligned}
\left(f^{p}\right)^{q c}(A) & =\inf _{\phi \in C_{0}^{\infty}\left(\mathbf{B} ; \mathbf{R}^{n}\right)} f_{\mathbf{B}} f^{p}(A+\nabla \phi) \\
& \geq \frac{|A|^{p-n}}{C_{f}(n, \delta)}\left[\left(l(p)^{-1}-\delta\right)|A|^{n}-n^{\frac{n}{2}} \operatorname{det} A\right] .
\end{aligned}
$$

Corollary 3.2. Let $f$ be as given in the theorem. Then $\mathcal{Z}\left(\left(f^{p}\right)^{q c}\right) \subseteq K_{l(p)}$ for all $p \in\left[p_{2}(n), n\right]$, where $l(p)$ is defined as in $(2.10)$. In particular, $\mathcal{Z}\left(\left(f^{n}\right)^{q c}\right)=K_{1}=$ $\mathcal{C}_{n}$

Proof. Suppose $\left(f^{p}\right)^{q c}(A)=0$. Then (3.3) implies $\left[l(p)^{-1}-\delta\right]|A|^{n} \leq n^{\frac{n}{2}} \operatorname{det} A$ for all $\delta>0$. Hence, letting $\delta \rightarrow 0^{+}$, we have $|A|^{n} \leq l(p) n^{\frac{n}{2}} \operatorname{det} A$, thus $A \in K_{l(p)}$.

In Theorem 3.1, choose $f=d_{\mathcal{C}_{n}}, A=0$ and $\delta=1 / 3$; then we recover a result proved earlier in Yan [18].

Corollary 3.3. There exists a constant $\Gamma_{n}$ such that for all $p_{2}(n) \leq p \leq n$ and all balls $\mathbf{B}$,

$$
\int_{\mathbf{B}}|\nabla \phi(x)|^{p} d x \leq \Gamma_{n} \int_{\mathbf{B}} d_{\mathcal{C}_{n}}^{p}(\nabla \phi(x)) d x, \quad \phi \in W_{0}^{1, p}\left(\mathbf{B} ; \mathbf{R}^{n}\right) .
$$

\section{EKELAND'S VARIATIONAL PRINCIPLE AND HIGHER REGULARITY}

In this section, we combine the Ekeland variational principle and the reverse Hölder inequality with increasing support to obtain a higher regularity for certain minimizing sequences of a conformal energy functional.

First of all, we state the following version of Ekeland's variational principle; see $[5]$ and [6, Thm 4.2]. 
Lemma 4.1. Let $(\mathcal{V}, d)$ be a complete metric space and $J: \mathcal{V} \rightarrow \mathbf{R} \cup\{+\infty\}$ a lower semicontinuous functional which is bounded below. Let $u_{j} \in \mathcal{V}$ be given such that

$$
J\left(u_{j}\right) \leq \inf _{v \in \mathcal{V}} J(v)+\frac{1}{2 j^{2}} .
$$

Then there exists $v_{j} \in \mathcal{V}$ such that $J\left(v_{j}\right) \leq J\left(u_{j}\right)$ and $d\left(v_{j}, u_{j}\right) \leq 1 / j$ and

$$
J\left(v_{j}\right)<J(w)+j^{-1} d\left(w, v_{j}\right) \quad \forall w \in \mathcal{V}, w \neq v_{j} .
$$

In what follows, let $p \in\left[p_{2}(n), n\right)$ and $A \in \mathcal{Z}\left(\left(d_{\mathcal{C}_{n}}^{p}\right)^{q c}\right)$, and $\mathbf{B}$ be the unit ball. Define

$$
\mathcal{V}=\left\{A x+\phi(x) \mid \phi \in W_{0}^{1,1}\left(\mathbf{B} ; \mathbf{R}^{n}\right)\right\}, \quad d(u, v)=\int_{\mathbf{B}}|\nabla u-\nabla v|, u, v \in \mathcal{V} .
$$

Then $(\mathcal{V}, d)$ is a complete metric space. Let $J_{p}: \mathcal{V} \rightarrow \mathbf{R} \cup\{+\infty\}$ be a functional defined by

$$
J_{p}(u)=\int_{\mathbf{B}} d_{\mathcal{C}_{n}}^{p}(\nabla u(x)) d x, \quad u \in \mathcal{V} .
$$

Then Fatou's lemma easily shows that $J_{p}$ is lower semicontinuous on $(\mathcal{V}, d)$. A technical advantage of using the distance function can be seen later. Since $A \in$ $\mathcal{Z}\left(\left(d_{\mathcal{C}_{n}}^{p}\right)^{q c}\right)$, we have a sequence $\left\{\phi_{j}\right\}$ in $C_{0}^{\infty}\left(\mathbf{B} ; \mathbf{R}^{n}\right)$ such that

$$
\int_{\mathbf{B}} d_{\mathcal{C}_{n}}^{p}\left(A+\nabla \phi_{j}(x)\right) d x \leq \frac{1}{2 j^{2}}, \quad \forall j=1,2, \cdots .
$$

Let $u_{j}(x)=A x+\phi_{j}(x)$. Then $u_{j} \in \mathcal{V}, J_{p}\left(u_{j}\right) \leq \frac{1}{2 j^{2}}$, and thus $\inf _{v \in \mathcal{V}} J_{p}(v)=0$. Hence, by Lemma 4.1 , there exist $v_{j} \in \mathcal{V}$ for $j=1,2, \ldots$ such that

$$
\begin{gathered}
\int_{\mathbf{B}} d_{\mathcal{C}_{n}}^{p}\left(\nabla v_{j}\right) \leq \frac{1}{2 j^{2}}, \quad \int_{\mathbf{B}}\left|\nabla u_{j}-\nabla v_{j}\right| \leq j^{-1}, \\
\int_{\mathbf{B}} d_{\mathcal{C}_{n}}^{p}\left(\nabla v_{j}\right) \leq \int_{\mathbf{B}} d_{\mathcal{C}_{n}}^{p}(\nabla w)+j^{-1} \int_{\mathbf{B}}\left|\nabla v_{j}-\nabla w\right|, \quad \forall w \in \mathcal{V} .
\end{gathered}
$$

With these at our disposal, we can prove the following result.

Proposition 4.2. Both $\left\{u_{j}\right\}$ and $\left\{v_{j}\right\}$ are bounded in $W^{1, p}\left(\mathbf{B} ; \mathbf{R}^{n}\right)$. If we assume they converge weakly in $W^{1, p}\left(\mathbf{B} ; \mathbf{R}^{n}\right)$, then the weak limits must be equal and belong to $W^{1, n}\left(\mathbf{B} ; \mathbf{R}^{n}\right)$.

Proof. The boundedness of both sequences and the coincidence of weak limits follow from (4.2) and Corollary 3.3. Let $u_{0}$ be the weak limit. Then from weak lower semicontinuity ([1], [3]),

$$
\int_{\mathbf{B}}\left(d_{\mathcal{C}_{n}}^{p}\right)^{q c}\left(\nabla u_{0}\right) \leq \lim _{j \rightarrow \infty} \int_{\mathbf{B}}\left(d_{\mathcal{C}_{n}}^{p}\right)^{q c}\left(\nabla u_{j}\right) \leq \lim _{j \rightarrow \infty} \int_{\mathbf{B}} d_{\mathcal{C}_{n}}^{p}\left(\nabla u_{j}\right)=0 .
$$

Thus, $\nabla u_{0}(x) \in \mathcal{Z}\left(\left(d_{\mathcal{C}_{n}}^{p}\right)^{q c}\right) \subseteq K_{l(p)}$. Since $p_{2}(n) \leq p \leq n$, Lemma 2.3 thus concludes $u_{0} \in W^{1, n}\left(\mathbf{B} ; \mathbf{R}^{n}\right)$.

We now use techniques of Caccioppoli-type estimates to derive the reverse Hölder inequality with increasing support. Before we proceed with this, several remarks are in order. First, since the integrand $d_{\mathcal{C}_{n}}^{p}$ of $J_{p}$ does not satisfy the usual growth condition as assumed in some standard Caccioppoli-type estimates ([8], [9]), we need our crucial coercivity estimates to obtain the desired reverse Hölder inequality with 
doubling supports. Moreover, we have to pay particular attention to the various constants involved to assure the uniform increment of the integrability.

Theorem 4.3. Let $A \in \mathcal{Z}\left(\left(d_{\mathcal{C}_{n}}^{p}\right)^{q c}\right)$ and $p_{2}(n) \leq p \leq n$, and let $u_{j}$ and $v_{j}$ be given as above. Then, for all balls $B_{2 R}=B(a, 2 R) \subset \subset \mathbf{B}$,

$$
f_{B_{R}}\left|\nabla v_{j}\right|^{p} \leq \beta_{n}\left(f_{B_{2 R}}\left|\nabla v_{j}\right|^{\frac{p n}{n+p}}\right)^{\frac{n+p}{n}}+\gamma_{n}, \quad \forall j \geq N_{n}
$$

where $\beta_{n}, \gamma_{n}$ and $N_{n}$ are constants depending only on the dimension $n$.

Proof. In what follows, we use $c_{0}, c_{1}, \ldots$ to denote the constants depending only on dimension $n$. Let $B_{2 R}=B(a, 2 R) \subset \subset \mathbf{B}$ and $\nu$ a number to be chosen later. Given $0<s_{1}<s_{2} \leq 2 R$, let $\eta \in C_{0}^{\infty}(\mathbf{B})$ be a cut-off function such that

$$
0 \leq \eta \leq 1,\left.\quad \eta\right|_{B_{s_{1}}}=1,\left.\quad \eta\right|_{\mathbf{B} \backslash B_{s_{2}}}=0, \quad|\nabla \eta| \leq c_{0}\left(s_{2}-s_{1}\right)^{-1} .
$$

Let $w=\eta \nu+(1-\eta) v_{j}$ and $\phi=v_{j}-w$. Then $w \in \mathcal{V}, \phi \in W_{0}^{1, p}\left(B_{s_{2}} ; \mathbf{R}^{n}\right)$ and

$$
\nabla w=(1-\eta) \nabla v_{j}-\left(v_{j}-\nu\right) \otimes \nabla \eta, \nabla \phi=\eta \nabla v_{j}+\left(v_{j}-\nu\right) \otimes \nabla \eta .
$$

By inequality $d_{\mathcal{K}}^{p}(X+Y) \leq 2^{n}\left(d_{\mathcal{K}}^{p}(X)+|Y|^{p}\right)$ and Corollary 3.3, we have

$$
\begin{aligned}
& \int_{B_{s_{1}}}\left|\nabla v_{j}\right|^{p} \leq \int_{B_{s_{2}}}|\nabla \phi|^{p} \leq \Gamma_{n} \int_{B_{s_{2}}} d_{\mathcal{C}_{n}}^{p}(\nabla \phi) \\
\leq & c_{1} \int_{B_{s_{2}}} d_{\mathcal{C}_{n}}^{p}\left(\nabla v_{j}\right)+\frac{c_{1}}{\left(s_{2}-s_{1}\right)^{p}} \int_{B_{s_{2}} \backslash B_{s_{1}}}\left|v_{j}-\nu\right|^{p} .
\end{aligned}
$$

Using (4.5), noting that $\nabla w=0$ on $B_{s_{1}}$ and $d_{\mathcal{C}_{n}}^{p}(X) \leq|X|^{p}$, we deduce

$$
\int_{B_{s_{2}}} d_{\mathcal{C}_{n}}^{p}(\nabla w) \leq c_{2} \int_{B_{s_{2}} \backslash B_{s_{1}}}\left|\nabla v_{j}\right|^{p}+\frac{c_{2}}{\left(s_{2}-s_{1}\right)^{p}} \int_{B_{s_{2}} \backslash B_{s_{1}}}\left|v_{j}-\nu\right|^{p} .
$$

Since $\nabla w=\nabla v_{j}$ in $\mathbf{B} \backslash B_{s_{2}}$, combining (4.3) and (4.5)-(4.7) gives

$$
\begin{gathered}
\int_{B_{s_{1}}}\left|\nabla v_{j}\right|^{p} \leq c_{3} \int_{B_{s_{2}} \backslash B_{s_{1}}}\left|\nabla v_{j}\right|^{p}+ \\
\frac{c_{3}}{\left(s_{2}-s_{1}\right)^{p}} \int_{B_{2 R}}\left|v_{j}-\nu\right|^{p}+\frac{c_{3}}{j} \int_{B_{s_{2}}}\left|\nabla v_{j}-\nabla w\right| .
\end{gathered}
$$

Let $N_{n}=2 c_{3}$ and $j \geq N_{n}$. Then by inequality $t \leq t^{p}+1$ for all $t \geq 0$ and $p \geq 1$,

$$
\int_{B_{s_{1}}}\left|\nabla v_{j}\right|^{p} \leq \frac{c_{4}}{1+c_{4}} \int_{B_{s_{2}}}\left|\nabla v_{j}\right|^{p}+\frac{c_{5}}{\left(s_{2}-s_{1}\right)^{p}} \int_{B_{2 R}}\left|v_{j}-\nu\right|^{p}+c_{6}\left|B_{2 R}\right| .
$$

Using this and the standard hole-filling arguments ([9, Lemma 5.1]), we have

$$
\int_{B_{R}}\left|\nabla v_{j}\right|^{p} \leq c_{7} R^{-p} \int_{B_{2 R}}\left|v_{j}-\nu\right|^{p}+c_{7}\left|B_{2 R}\right|
$$

which yields

$$
f_{B_{R}}\left|\nabla v_{j}\right|^{p} \leq \frac{c_{8}}{R^{n+p}} \int_{B_{2 R}}\left|v_{j}-\nu\right|^{p}+c_{8}
$$


Now let $\nu=\nu_{R}=f_{B_{2 R}} v_{j}$ and use the Sobolev-Poincaré inequality

$$
\int_{B_{2 R}}\left|v_{j}-\nu_{R}\right|^{p} \leq \sigma_{n}\left(\int_{B_{2 R}}\left|\nabla v_{j}\right|^{\frac{p n}{n+p}}\right)^{\frac{n+p}{n}}
$$

in (4.9), we obtain (4.4). The theorem is thus proved.

Theorem 4.4. There exists $\epsilon_{n}>0$ depending only on $n$ such that for any $p \in$ $\left[p_{2}(n), n\right], A \in \mathcal{Z}\left(\left(d_{\mathcal{C}_{n}}^{p}\right)^{q c}\right)$ and sequences $\left\{u_{j}\right\}$ and $\left\{v_{j}\right\}$ defined as above, one has

$$
\int_{\Omega}\left|\nabla v_{j}\right|^{p+\epsilon_{n}} \leq M_{\Omega}<\infty \quad \text { for all } j \geq N_{n} \text { and } \Omega \subset \subset \mathbf{B},
$$

where $N_{n}$ is the constant in the previous theorem.

Proof. For $j \geq N_{n}$, let $f_{j}=1+\left|\nabla v_{j}\right|^{\frac{p n}{n+p}}$ and $r=\frac{n+p}{n}$. By Proposition 4.2 and Theorem 4.3, $\left\{f_{j}\right\}$ is bounded in $L^{r}(\mathbf{B})$ and

$$
f_{B_{R}} f_{j}^{r} \leq \beta_{n}^{\prime}\left(f_{B_{2 R}} f_{j}\right)^{r} \quad \forall B_{2 R}=B(a, 2 R) \subset \subset \mathbf{B} .
$$

Therefore, by an improved version [9, Thm 6.1] of Gehring's Lemma [7, Lemma 2] on reverse Hölder inequality, we have $\left\{f_{j}\right\}$ is (locally) bounded in $L_{l o c}^{r^{\prime}}(\mathbf{B})$ for some $r^{\prime}>r$, thus $\left\{v_{j}\right\}$ is (locally) bounded in $W_{l o c}^{1, p+\epsilon}(\mathbf{B})$ for some $\epsilon>0$. Furthermore, from the proof of [9, Thm 6.1], one sees that the increment $\epsilon>0$ can be chosen uniformly for $p \in\left[p_{2}(n), n\right]$.

\section{Proof of the main Result: Theorem B}

Let $p \in\left[p_{2}(n), n\right]$. We shall prove $\mathcal{Z}\left(\left(d_{\mathcal{C}_{n}}^{p}\right)^{q c}\right)=\mathcal{C}_{n}$; thus $\mathbf{Q}_{p}\left(\mathcal{C}_{n}\right)=\mathcal{C}_{n}$. Suppose $A \in \mathcal{Z}\left(\left(d_{\mathcal{C}_{n}}^{p}\right)^{q c}\right)$. Let $\left\{u_{j}\right\}$ and $\left\{v_{j}\right\}$ be defined as before and let $u_{0}$ be the weak limit in Proposition 4.2. Then $v_{j} \rightarrow u_{0}$ in $W_{l o c}^{1, p+\epsilon_{n}}\left(\mathbf{B} ; \mathbf{R}^{n}\right)$, where $\epsilon_{n}>0$ is the constant determined in Theorem 4.4. Let $s=p+\frac{\epsilon_{n}}{2}$; then inequality $d_{\mathcal{C}_{n}}^{s}(X) \leq$ $\delta|X|^{p+\epsilon_{n}}+C(\delta) d_{\mathcal{C}_{n}}^{p}(X)$ and Theorem 4.4 imply

$$
\int_{\Omega} d_{\mathcal{C}_{n}}^{s}\left(\nabla v_{j}\right) \leq \delta \int_{\Omega}\left|\nabla v_{j}\right|^{p+\epsilon_{n}}+C(\delta) \int_{\Omega} d_{\mathcal{C}_{n}}^{p}\left(\nabla v_{j}\right) \leq \delta M_{\Omega}+\frac{1}{2 j^{2}} C(\delta)
$$

for all $\Omega \subset \subset \mathbf{B}$. Let first $j \rightarrow \infty$ and then $\delta \rightarrow 0$; we have $\int_{\Omega} d_{\mathcal{C}_{n}}^{s}\left(\nabla v_{j}\right) \rightarrow 0$ which, by $v_{j} \rightarrow u_{0}$ in $W^{1, p+\epsilon_{n}}\left(\Omega ; \mathbf{R}^{n}\right)$, implies

$$
\int_{\Omega}\left(d_{\mathcal{C}_{n}}^{s}\right)^{q c}\left(\nabla u_{0}\right) \leq \lim _{j \rightarrow \infty} \int_{\Omega} d_{\mathcal{C}_{n}}^{s}\left(\nabla v_{j}\right)=0
$$

Since $\Omega \subset \subset \mathbf{B}$ is arbitrary, so $\int_{\mathbf{B}}\left(d_{\mathcal{C}_{n}}^{s}\right)^{q c}\left(\nabla u_{0}\right)=0$. Note that $u_{0} \in W^{1, n}\left(\mathbf{B} ; \mathbf{R}^{n}\right)$ and $u_{0}(x)=A x$ on $\partial \mathbf{B}$. A simple approximation argument shows

$$
\left(d_{\mathcal{C}_{n}}^{s}\right)^{q c}(A) \leq f_{\mathbf{B}}\left(d_{\mathcal{C}_{n}}^{s}\right)^{q c}\left(\nabla u_{0}(x)\right) d x=0
$$

hence, $A \in \mathcal{Z}\left(\left(d_{\mathcal{C}_{n}}^{s}\right)^{q c}\right)$ for $s=p+\frac{\epsilon_{n}}{2}$. Repeating this procedure finitely many times, we eventually obtain $A \in \mathcal{Z}\left(\left(d_{\mathcal{C}_{n}}^{n}\right)^{q c}\right)$; hence by Corollary 3.2

$$
\mathcal{Z}\left(\left(d_{\mathcal{C}_{n}}^{p}\right)^{q c}\right)=\mathcal{Z}\left(\left(d_{\mathcal{C}_{n}}^{n}\right)^{q c}\right)=K_{1}=\mathcal{C}_{n}
$$

as desired. Theorem B is thus proved. 


\section{ACKNOWLEDGMENTS}

The first author would like to acknowledge the stimulating discussions with Stefan Müller and Vladimir Šverák, and he is also grateful to Tadeusz Iwaniec for his interest and for sending many references.

\section{REFERENCES}

1. Acerbi, E. and Fusco, N., Semicontinuity problems in the calculus of variations, Arch. Rational Mech. Anal., 86 (1984), 125-145. MR 85m:49021

2. Ball, J. M., Convexity conditions and existence theorems in nonlinear elasticity, Arch. Rational Mech. Anal., 63 (1977), 337-403. MR 57:14788

3. Ball, J. M. and Murat, F., $W^{1, p}$-Quasiconvexity and variational problems for multiple integrals, J. Funct. Anal., 58 (1984), 225-253. MR 87g:49011a

4. Dacorogna, B., "Direct Methods in the Calculus of Variations," Springer-Verlag, Berlin, Heidelberg, New York, 1989. MR 90e:49001

5. Ekeland, I., On the variational principle, J. Math. Anal. Appl., 47 (1974), 324-353. MR 49:11344

6. De Figueiredo, D. G., "The Ekeland Variational Principle with Applications and Detours," Tata Institute Lecture, Springer-Verlag, Berlin, Heidelberg, New York, 1989.

7. Gehring, F. W., The $L^{p}$-integrability of the partial derivatives of a quasiconformal mapping, Acta Math., 130 (1973), 265-277. MR 53:5861

8. Giaquinta, M., "Multiple Integrals in the Calculus of Variations and Nonlinear Elliptic Systems," Princeton University Press, Princeton, 1983. MR 86b:49003

9. Giaquinta, M., "Introduction to Regularity Theory for Nonlinear Elliptic Systems," Lectures in Mathematics, ETH Zürich, Birkhäuser, Basel, Berlin, 1993. MR 94g:49002

10. Greco, L. and Iwaniec, T., New inequalities for the Jacobian, Ann. Inst. H. Poincaré, Analyse non linéaire, 11(1) (1994), 17-35. MR 95b:42020

11. Iwaniec, T., p-Harmonic tensors and quasiregular mappings, Ann. Math., 136 (1992), 589624. MR 94d:30034

12. Iwaniec, T. and Martin, G., Quasiregular mappings in even dimensions, Acta Math., 170 (1993), 29-81. MR 94m:30046

13. Iwaniec, T. and Sbordone, C., Weak minima of variational integrals, J. Reine Angew. Math., 454 (1994), 143-161. MR 95d:49035

14. Morrey, C. B. Jr., "Multiple Integrals in the Calculus of Variations," Springer-Verlag, Berlin, Heidelberg, New York, 1966. MR 34:2380

15. Müller, S., Šverák, V. and Yan, B., Sharp stability results for almost conformal maps in even dimensions, submitted to Journ. Geom. Analysis.

16. Reshetnyak, Yu. G., "Space Mappings with Bounded Distortion," Transl. Math. Mono., A.M.S., Vol. 73, 1989. MR 90d:30067

17. Šverák, V., Lower semicontinuity for variational integral functionals and compensated compactness, Proceedings of I.C.M., Zürich, 1994.

18. Yan, B., Remarks about $W^{1, p}$-stability of the conformal set in higher dimensions, Ann. Inst. H. Poincaré, Analyse non linéaire, 13 (6), 1996.

19. Yan, B., On rank-one convex and polyconvex conformal energy functions with slow growth, Proc. Roy. Soc. Edinb., Ser. A. (to appear)

20. Yan, B., On $W^{1, p}$-quasiconvex hull of sets of matrices and weak convergence in Sobolev spaces, preprint, 1995.

21. Zhang, K., A construction of quasiconvex functions with linear growth at infinity, Ann. Scuola Norm. Sup. Pisa, 19(3) (1992), 313-326. MR 94d:49018

Department of Mathematics, Michigan State University, East Lansing, Michigan 48824

E-mail address: yan@math.msu.edu

E-mail address: zf zhou@math.msu.edu 